\title{
Adiabaticity parameters for the categorization of light-matter interaction - from weak to strong driving
}

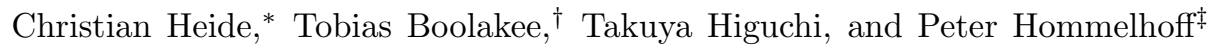 \\ Laser Physics, Department of Physics, Friedrich-Alexander-Universität \\ Erlangen-Nürnberg (FAU), Staudtstrasse 1, D-91058 Erlangen, Germany
}

(Dated: April 22, 2021)

\begin{abstract}
We investigate theoretically and numerically the light-matter interaction in a two-level system (TLS) as a model system for excitation in a solid-state band structure. We identify five clearly distinct excitation regimes, categorized with well known adiabaticity parameters: (1) the perturbative multiphoton absorption regime for small driving field strengths, and four light field-driven regimes, where intraband motion connects different TLS: (2) the impulsive Landau-Zener (LZ) regime, (3) the non-impulsive LZ regime, (4) the adiabatic regime and (5) the adiabatic-impulsive regime for large electric field strengths. This categorization is tremendously helpful to understand the highly complex excitation dynamics in any TLS, in particular when the driving field strength varies, and naturally connects Rabi physics with Landau-Zener physics. In addition, we find an insightful analytical expression for the photon orders connecting the perturbative multiphoton regime with the light field-driven regimes. Moreover, in the adiabatic-impulsive regime, adiabatic motion and impulsive LZ transitions are equally important, leading to an inversion symmetry breaking of the TLS when applying few cycle laser pulses. This categorization allows a deep understanding of driven TLS in a large variety of settings ranging from cold atoms and molecules to solids and qubits, and will help to find optimal driving parameters for a given purpose.
\end{abstract}

The interaction between intense optical fields and two-level systems (TLS) has facilitated controlling electrons coherently on ultrashort timescales. This gave rise to new research areas including the efficient generation of high-harmonics in atoms and solids [1 4, light field-driven ionization and electron emission 5 as well as light field-driven current generation in solids 6] 8]. Simultaneously, studying light-matter interaction in engineered electrodynamic environments led to the vibrant field of cavity quantum electrodynamics (QED) where recent systems based on superconducting qubits, exciton and intersubband polaritons and electron cyclotron resonance are used to explore their peculiar properties in the regimes of ultrastrong and deep strong coupling [9, 10. Likewise, coherent electron dynamics is investigated in qubits for information processing [11, 12] and, more recently, in topologically relevant materials [13 16. In all these cases, the underlying physics can be simplified, categorized and described with a TLS interacting with light. Here, we provide a clear categorization of different excitation regimes using well-known adiabaticity parameters, with a focus on solids. Finally we show that the presented categorization is applicable to a multitude of different systems.

As long as the light-field is weak, momentum exchange between light and electrons, i.e., intraband motion, can be neglected, and the occupation of the bands is well described with resonant Rabi physics, with interband transitions only [17, 18. This picture, however, becomes unsuited or at least difficult to interpret when the

\footnotetext{
* Christian.Heide@fau.de These two authors contributed equally

$\dagger$ Tobias.Boolakee@fau.de These two authors contributed equally $\ddagger$ Peter.Hommelhoff@fau.de
}

light-field becomes so strong that different electron wave numbers become coupled resulting in field-driven intraband motion, which may influences interband transitions. In this case, the electron dynamics is better described as (repeated) Landau-Zener transitions between valence and conduction band [6], 19,27]. An appropriate basis to describe intraband motion (coupling of different k-states) and interband transitions is the Houston basis [8, 25].

Whereas pure diabatic interband or adiabatic intraband electron dynamics have been well investigated in the context of strong-field physics, such as with high harmonic generation (HHG) in solids [1, 3, 13, 26, 28, 33, intraband motion and interband transitions acting in a combined fashion have been investigated to a less comprehensive degree. Based on adiabaticity parameters, we identify a novel regime of light-matter interaction, where both intraband motion and interband transitions generate an off-resonant, residual excitation. In contrast to HHG, which is not able to probe this off-resonant excitation directly, it can be well observed as a residual current 6 . With this new regime, we can now give a complete and a general picture of TLS physics and can categorize it into five clearly distinct regimes.

In his seminal paper, Keldysh introduced the adiabaticity parameter $\gamma \equiv \omega \sqrt{m \Delta} /\left(e E_{0}\right)$ for electrons in a TLS 34 . Here, $\omega$ is the driving frequency of the light, $E_{0}$ its peak electric field strength, $m$ the effective electron mass, $\Delta$ the minimal energy spacing between two bands and $e$ the electron charge. Assuming monochromatic light and excitation at $\Delta$, we can express the Keldysh parameter as

$$
\gamma=\frac{\Delta}{2 \hbar \Omega_{\mathrm{R}}}
$$




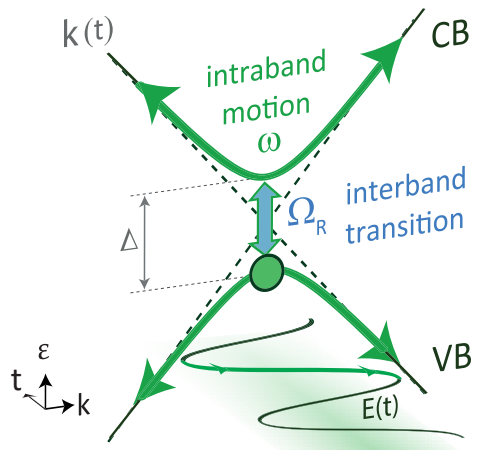

FIG. 1. Light-field driven electron dynamics in a two-level system. When the electric field is small, intraband motion can be neglected and the excitation oscillates resonantly between valence (VB) and conduction band (CB) with the Rabi frequency $\Omega_{R}$. When the electric field becomes strong, the Rabi frequency exceeds the driving frequency, and intraband motion strongly influences interband transitions.

with $\Omega_{\mathrm{R}}=v_{\mathrm{F}} e E_{0} /(\hbar \omega)$ the Rabi frequency and $v_{\mathrm{F}}$ the Fermi velocity, see Fig.1 and SI for a detailed derivation. When $2 \hbar \Omega_{\mathrm{R}}$ exceeds $\Delta, \gamma$ becomes smaller than 1 and the light-matter interaction enters the well-known strong-field regime.

In case of resonant excitation, $\Delta=\hbar \omega$, we obtain the resonant adiabaticity parameter from Eq. (1) [25, 35, 36. as

$$
z_{\mathcal{R}}=\frac{2 \Omega_{\mathrm{R}}}{\omega} .
$$

Here, $2 \Omega_{\mathrm{R}}$ can be interpreted as the inverse of the transition time $\tau_{t}\left(2 \Omega_{\mathrm{R}}=(2 \pi) / \tau_{t}\right)$ from the valence to the conduction band. Figure 1 shows schematically the competition of Rabi oscillations with $\Omega_{\mathrm{R}}$ (blue arrow) and intraband motion, driven with $\omega$ (thick green arrows). When $\tau_{t}$ becomes shorter than the driving period of the light, $z_{\mathcal{R}}$ becomes larger than 1 . In that regime, colloquially speaking, the electron has enough time to undergo a transition from one to the other band within an optical cycle.

We will continue with further insightful parameters later but now first model the light-matter interaction by solving the time-dependent Schrödinger equation numerically. The resulting excitation maps will help us to identify the various regimes. We consider a TLS with two energy levels $\pm \Delta / 2$ and a time-dependent perturbation $\pm \alpha(t) / 2$, representing an avoided crossing [19, 21, 37,39]. The Hamiltonian of this system reads:

$$
\hat{\mathcal{H}}(t)=-\frac{\alpha(t)}{2} \hat{\sigma}_{\mathrm{x}}-\frac{\Delta}{2} \hat{\sigma}_{\mathrm{z}},
$$

with $\hat{\sigma}_{\mathrm{x}, \mathrm{z}}$ the Pauli matrices. The eigenenergies are $\varepsilon_{ \pm}(t) \equiv \pm \frac{1}{2} \varepsilon(t)$, where $\varepsilon(t)=\sqrt{\Delta^{2}+\alpha(t)^{2}}$ is the timedependent energy difference between the two levels. By taking $k(t)$ as the time-dependent wave number of an electron, and $\pm v_{\mathrm{F}}$ as the slopes of the two crossing levels, Eq. (3) with $\alpha(t)=2 \hbar v_{\mathrm{F}} k(t)$ represents a solid state band structure with $\varepsilon_{ \pm}(k)$ the conduction band $(+)$ and valence band energy $(-)$ (and $\Delta$ the band gap). We assume $v_{\mathrm{F}}=1 \mathrm{~nm} / \mathrm{fs}$. The change of the electron wave number due to the electric field is described by the Bloch acceleration theorem $\dot{k}(t)=-(e / \hbar) E(t)$ [7, 8, 40, 41]. We note that when the electric field is weak, the electron dynamics is well described with a trivial TLS of fixed levels $\varepsilon_{ \pm}$. However, when the electric field becomes large, different $k$-values become coupled and the electron undergoes intraband motion, probing the band structure. For even larger electric field strengths (i.e., $\gamma<0.01$ ), relativistic effects may become important, which are beyond the presented categorization (see SI).

In the simulations we apply a linearly polarized vector potential

$$
A(t)=-E_{0} / \omega \exp \left(-2 \ln 2\left(t / \tau_{p}\right)^{2}\right) \sin \left(\omega t+\phi_{\mathrm{CEP}}\right),
$$

associated with an electric field $E(t)=-\dot{A}(t)$, to model the temporal evolution of the conduction band population. By defining the vector potential we satisfy $A(-\infty)=A(\infty)$ and thus omit dc components in the electric field. A pulse duration of $\tau_{p}=5 \mathrm{fs}$ is chosen with central photon energy $\hbar \omega=1.55 \mathrm{eV}$ and a carrierenvelope phase $\phi_{\mathrm{CEP}}=\pi / 2$. For simplicity, we first consider electrons with an initial wave number of $k_{0}=0$. Note that while different pulse durations, dephasing and dispersion effects may change the population distribution, the presented categorization remains valid (see SI).

Figures 2(a-e) show in the lower panels the conduction band population $\rho^{\mathrm{CB}}$ as a function of time for various regimes, discussed in detail in what follows. In Fig.2(f), we show the residual conduction band population parameterized by $\gamma$ and the multi-photon parameter $M=\Delta /(\hbar \omega)$, measuring the band gap in units of the photon energy. Based on $\gamma$ and $z_{\mathcal{R}}$ we can now identify and categorize various regimes, which show an entirely different temporal evolution of the conduction band population [Fig.2 (a-e)].

(1) Perturbative multi-photon absorption regime: When the electric field is weak $(\gamma>1)$, population is found at $M \approx 1$, reflecting resonant excitation [Fig.2](f, $\mathrm{g})$ ]. Here, the population $\rho^{\mathrm{CB}}$ rises during the laser pulse gradually [Fig.2[(a)]. The red lines, horizontal for $\gamma>1$, represent the resonance condition: $M$ is integer. The population width along $M$ reflects the spectral width of the laser pulse. Increasing the electric field strength (decreasing $\gamma$ ) results in an increase of $\Omega_{\mathrm{R}} \propto E_{0}$, hence Rabi oscillations become visible.

Transition to the strong-field regime (2)-(5): Around $\gamma \approx 1$, the interaction strength $2 \hbar \Omega_{\mathrm{R}}$ is of the order of the band gap [Eq. (1)] and light field-driven intraband motion cannot be neglected anymore. Hence, intraband motion leads to a variation of $\varepsilon(t)$, which in turn results to a rotation of the features in Fig.2(f). 


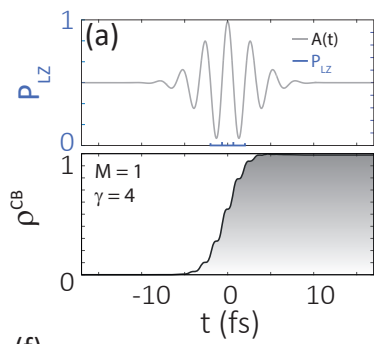

(f)
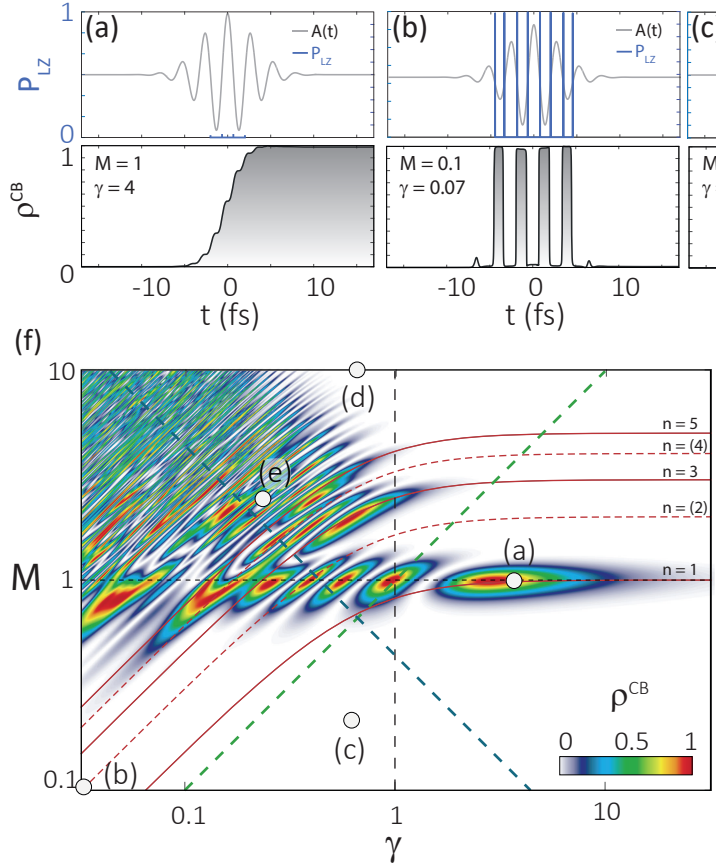

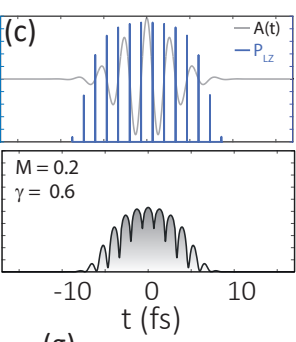

(g)
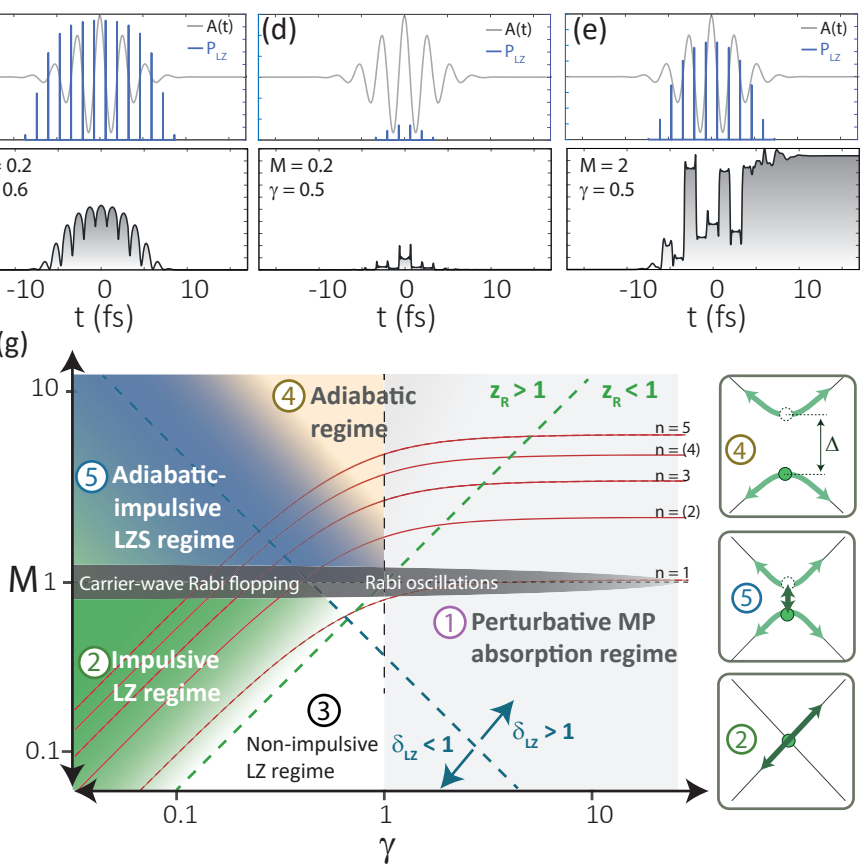

FIG. 2. Regimes of a light field-driven two-level system. (a-e) Temporal evolution of the conduction band population $\rho^{\mathrm{CB}}$ for a Gaussian pulse $\left(\tau_{\mathrm{P}}=5 \mathrm{fs}, \phi_{\mathrm{CEP}}=\pi / 2\right)$ with electrons starting from $k_{0}=0$ from our TDSE model. The top panels show the normalized vector potential $A(t)$ (grey) and $P_{\mathrm{LZ}}$ (blue) for an LZ transition at $k_{0}$. The laser and system parameters are identified by $\gamma$ and $M$, given in each legend. (f) Map of the residual conduction band population. The filled spheres relate to the panels (a-e). See text for details. (g) same as (f), with various regimes indicated: (1) Perturbative MP absorption regime, (2) impulsive LZ regime, (3) non-impulsive LZ regime, (4) adiabatic regime and (5) adiabatic-impulsive regime. Whereas for $\gamma>1$ only perturbative, resonant one-photon $(n=1)$ absorption at $M=1$ is found, for $\gamma<1$ off-resonant excitation occurs. In particular for $P_{\mathrm{LZ}} \approx 0.5, \delta_{\mathrm{LZ}} \approx \ln (2) /(2 \pi)$ and $z_{\mathcal{R}}>1$, defining the adiabatic-impulsive regime (5), most off-resonant excitation is found. The red lines represent odd (solid) and even (dashed) resonances for the light matter interaction, based on Eq. (6). The boxes relate to three regimes as indicated by the number. Note the dashed lines showing the crossing areas for the various adiabaticity parameters.

For $\gamma<1$, carrier-wave Rabi flopping occurs and the horizontal lines given by multiples of the photon energy fail as a valid quantity to specify resonant absorption, reflecting the AC Stark effect 23 . To extend the resonance condition to this field-driven regime, we calculate the dynamical phase

$$
\phi=\frac{1}{\hbar} \int \varepsilon(t) \mathrm{d} t
$$

When a phase of $\phi=2 \pi$ is accumulated within an optical cycle of the laser pulse, population at the next higher multi-photon resonance $(n)$ is found 23 . For monochromatic excitation, we analytically obtain the resonance condition

$$
M=\frac{n \pi}{2 \mathfrak{e}_{l}\left(-\gamma^{-2}\right)} .
$$

Here, $\mathfrak{e}_{l}$ denotes the complete elliptic integral of the second kind and $n$ the order of the resonance (see SI). We see that when $\gamma$ decreases, the photon resonances $n$ no longer match integer multiples of $M$ but are shifted towards smaller $M$. Equation (6) is plotted as red solid lines in Fig.2(f), for various $n$, perfectly matching the conduction band population obtained from the TDSE simulation. When $\gamma \gg 1$, the elliptic integral becomes $\pi / 2$ and the photon resonances are found at integer multiples of the band gap, i.e., $M=n$, as expected for weak fields. In contrast, at $\gamma \approx 0.4$ the intraband motion becomes so strong that $n=2 M$ and, thus, the two-photon resonance is found at $M=1$. We note that due to the inversion symmetry of the TLS at $k_{0}=0$ population only arises for odd photon orders [see dashed lines for even orders in Fig. 2(f)].

In the field-driven regime, i.e., $\gamma<1$ it is helpful to describe the electron dynamics within the Landau-Zener (LZ) formalism, see SI and 19, 21, 22, 42, 43. At time $t^{\prime}$, when the electron reaches the minimal separation of valence and conduction band, the transition from one to the other band takes the form of an LZ transition. The transition probability is approximated by the famous Landau-Zener formula

$$
P_{\mathrm{LZ}}=\exp \left(-2 \pi \delta_{\mathrm{LZ}}\right)
$$


with

$$
\delta_{\mathrm{LZ}} \equiv \frac{1}{\hbar \alpha\left(t^{\prime}\right)}\left(\frac{\Delta}{2}\right)^{2}
$$

the Landau-Zener adiabaticity parameter [21, 37, 38. Within the field-driven regime we now find four categories of electron dynamics.

(2) Impulsive Landau-Zener regime: When $\delta_{\mathrm{LZ}} \ll 1$ and $z_{\mathcal{R}}>1$ the electron undergoes a sequence of fast LZ transitions with probability $P_{\mathrm{LZ}}$ close to unity [Fig. 2(b)]. Within one optical cycle the electron experiences a transition from the valence to the conduction band and back to the valence band. Because of $z_{\mathcal{R}}>1$, the regime is called impulsive. After the laser pulse, the electron ends up in the initial band, hence no excitation is found [Fig.2[(f, g)].

(3) Non-impulsive Landau-Zener regime: When the transition time $\tau_{t}$ is longer than the optical period (i.e., $z_{\mathcal{R}}<1$ ), the LZ transition can no longer be considered impulsive. Figure 2(c) shows that even when the LZ probability is large (blue lines in top panel) there is not enough time for an efficient electron excitation. Also here, the conduction band is not populated after the laser pulse is gone, albeit for very different reasons than in (2) [Fig.2(f)].

(4) Adiabatic regime: When $\delta_{\mathrm{LZ}} \gg 1$, the probability for an electron to undergo an LZ transition approaches zero and, thus, the electron undergoes pure intraband motion [Fig.2(d)]. Yet, we note that the intraband motion during the laser pulse is a well-investigated source of intraband HHG [29, 44].

(5) Adiabatic-impulsive Landau-ZenerStückelberg regime: When $\delta_{\mathrm{LZ}} \approx \ln (2) /(2 \pi)$, $P_{\mathrm{LZ}} \approx 0.5$. Hence after one LZ transition event, the electron wave function is equally split into valence and conduction band, so a part of the electron wave function undergoes an LZ transition, while the remainder stays adiabatically in the valence band [Fig.22(e)]. Due to the oscillatory nature of the driving, this happens periodically with every half cycle of the laser pulse. Interference of the electronic wave function components, each with a different accumulated phase $\phi$ [Eq. [25]], determines the conduction band excitation probability. We observe that, intriguingly, the net excitation probability is highest in this regime. We note that repeated coherent LZ transitions is called Landau-Zener-Stückelberg interference [19, 21, 37, 39].

So far, we have discussed electrons starting from $k_{0}=0$ only. Electrons with $k_{0}>0$ and $k_{0}<0$ can experience different dynamics, in particular, when a pulse with a broken time inversion symmetry $(E(-t) \neq E(t))$ is applied, e.g., one with $\phi_{\mathrm{CEP}}= \pm \pi / 2$ [45. Figure3 (a) shows schematically the electron trajectory in reciprocal space for an electron starting at $k_{0}>0$ (green line) and $k_{0}<0$ (blue line). For $\phi_{\mathrm{CEP}}= \pm \pi / 2$, the number of $\mathrm{LZ}$ transitions and their temporal spacing between an electron starting at initially positive or negative wave number
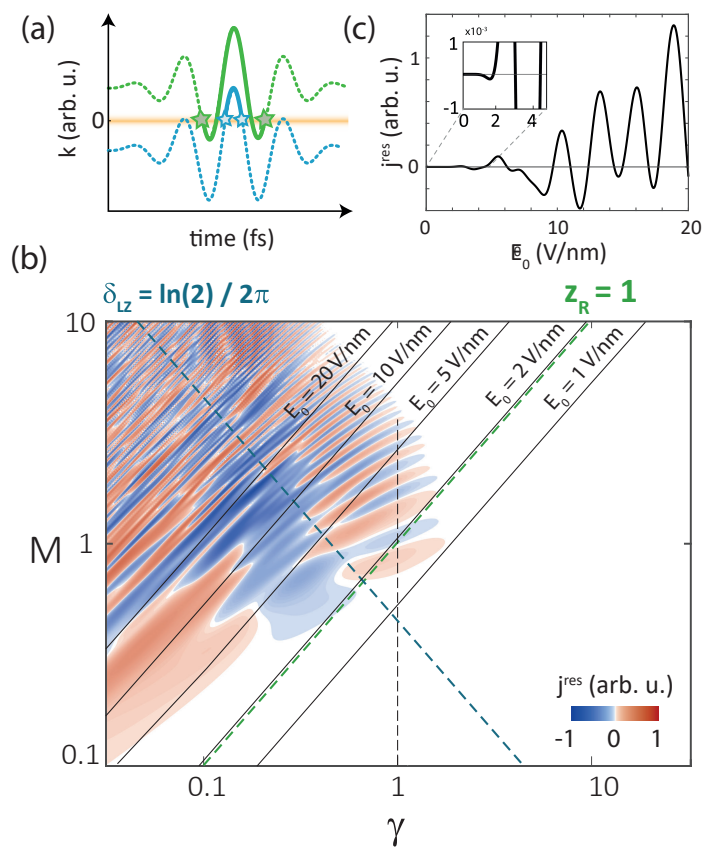

FIG. 3. (a) Temporal evolution of the electron trajectory for an electron with initially positive (green line) and negative (blue line) wave number. The orange line indicates the region of high LZ transition probability. For a field waveform with broken time inversion symmetry, the two electron trajectories can experience a different number of LZ transitions and/or a different total quantum mechanical phase evolution, in particular when, the length of the trajectory (green and blue) is different. (b) Residual electric current as function of $\gamma$ and $M$ calculated with the TDSE model. The maximal residual current is found in the adiabatic-impulsive LZS regime. The black lines indicate iso-lines for constant $E_{0}$. (c) Integrated current along lines with constant $E_{0}$, as a function of $E_{0}$. The oscillatory nature of $j^{\text {res }}\left(E_{0}\right)$ is a result of the wave numberdependent quantum mechanical phase evolution.

may differ, resulting in asymmetric residual population. As a consequence, a nonzero residual ballistic current

$$
j^{\mathrm{res}}=g_{s} e \sum_{m=\mathrm{CB}, \mathrm{VB}} \int_{-\infty}^{\infty} v^{(m)}(k(t)) \rho^{(m)}\left(k_{0}, t\right) \frac{\mathrm{d} k}{2 \pi},
$$

with $v^{(m)}(k)=\hbar^{-1} \frac{\partial \varepsilon_{ \pm}(k)}{\partial k}$ is generated. The factor $g_{s}=2$ accounts for two kinds of spins.

In Fig. 3(b) we show the map of the residual current density, taking all initial $k$-values into account. With the help of the above, we can now understand the intricate pattern of the current map. Residual current is mainly found in the adiabatic-impulsive LZS regime. Increasing the electric field strength, i.e., decreasing $\gamma$, starting at $\gamma \approx 1$, results in an increase of the accumulated dynamical phase, causing more and more current reversals as a function of $E_{0}$. Iso-lines for constant electric field strengths $E_{0}=1 \ldots 20 \mathrm{~V} / \mathrm{nm}$ are drawn as solid black lines. Around $E_{0}=1 \mathrm{~V} / \mathrm{nm}$ almost no residual current is obtained. Increasing the electric field strength towards 
$2 \mathrm{~V} / \mathrm{nm}$ results in nonzero $j^{\text {res }}$, at around $2 \mathrm{~V} / \mathrm{nm}$. This is shown and continued in the integrated current, along constant field strength in Fig.3(c). The oscillatory nature of the current as a function of the electric field strength reflects Landau-Zener-Stückelberg interference with varying accumulated phase, as also discussed above.

The current map obtained here numerically also matches an important previous result: In 8 it was shown that the CEP-dependent photocurrent switches sign at a field strength of $1.8 \mathrm{~V} / \mathrm{nm}$, perfectly coinciding with the first current reversal shown in Fig. 3. (c). Intriguingly, the adiabaticity parameters here are $\gamma<1$ and $z_{\mathcal{R}}>1$, indicating that the adiabatic-impulsive LZS regime has been entered in experiment.

Our TLS numerical results are based on the example of a narrow-band gap band structure to provide a connection to experimental conditions as given by strong-field physics in solids. However, the discussion is applicable to any avoided level crossing by appropriate transformation of parameters. For example, in wide-band gap systems, the Bloch frequency rather than the Rabi frequency is used to define $z_{\mathcal{R}}$ [25. Here, Wannier-Stark (WS) localization and Bloch oscillations are the dominating mechanism for LZ transitions [6].

In cavity QED, the characteristic system coupling $g$ is compared with $\omega$ to categorize excitation regimes [9, 10]. In particular, in the ultrastrong coupling regime $(g / \omega>$
$0.1)$ and the deep strong coupling regime $(g / \omega>1)$, the rotating wave approximation breaks down and new quantum phenomena emerge [46]. In full analogy to $g / \omega$, we distinguish between perturbative Rabi physics and the non-impulsive LZ regime for $z_{\mathcal{R}}<1$ and the impulsive strong-field regimes for $z_{\mathcal{R}}>1$.

In summary, we have presented five different regimes of light-matter interaction in a two-level system, categorized by adiabaticity parameters. This way, we can understand the intricate dynamics of each regime by delimiting it from but also connecting it to its neighboring regimes. Although we have focused on electrons in a solid state band structure, the here discussed dynamics and categorization into different regimes is well applicable to a large variety of two-level systems, which may include or give rise to nonadiabatic multielectron dynamics [43, 47, conical intersections [48, 49, Wannier-Stark localization [6, 50, cavity QED [9, 10], and the Kibble-Zurek mechanism [51, for which the time-dependent perturbation $\alpha(t)$ needs to be adapted appropriately, while the rest of the model is unchanged. Specifically, 2D materials can be well approximated as two-level systems [8, 52, 53]. Furthermore, our work is directly relevant to quantum information processing [11, 19, 54, where coherent electronics based on LZS interference has been recently implemented [12]. We expect that the presented categorization will help to understand fundamental yet complex light-matter interaction results on a new level across a large variety of systems.

\section{Appendix}

\section{A. General form of a two-level system}

We consider a two-level system (TLS) with two constant energy levels $\pm \Delta / 2$ and a time-dependent energy perturbation $\alpha(t) / 2$ acting on them, i.e., time-dependent coupling between energy levels. Such a system is described by the Hamiltonian

$$
\begin{aligned}
\hat{\mathcal{H}}(t) & =\hat{\mathcal{H}}_{0}+\hat{\mathcal{H}}_{\text {int }}(t) \\
& =-\frac{1}{2}\left(\begin{array}{cc}
\Delta & \alpha(t) \\
\alpha(t) & -\Delta
\end{array}\right) .
\end{aligned}
$$

Here, $\hat{\mathcal{H}}_{0}=-\frac{\Delta}{2} \hat{\sigma}_{\mathrm{z}}$ represents the bare two-level system with two eigenvalues $\pm \Delta / 2$ and $\hat{\mathcal{H}}_{\text {int }}(t)=-\frac{\alpha(t)}{2} \hat{\sigma}_{\mathrm{x}}$ the interaction Hamiltonian, describing how the system evolves under a time-dependent perturbation. $\hat{\sigma}_{\mathrm{x}}$ and $\hat{\sigma}_{\mathrm{z}}$ are Pauli matrices. By diagonalizing $\hat{\mathcal{H}}(t)$, one obtains the instantaneous eigenvalues

$$
\varepsilon(t)_{ \pm} \equiv \pm \frac{1}{2} \sqrt{\Delta^{2}+\alpha(t)^{2}}= \pm \frac{1}{2} \varepsilon(t) .
$$

Hereby,

$$
\varepsilon(t)=\sqrt{\Delta^{2}+\alpha(t)^{2}}
$$

is the time-dependent energy difference between the two energy states $\varepsilon_{+}$and $\varepsilon_{-}$, as depicted in Fig. 4 .

\section{B. Solid-state two-level system}

One example of a TLS system can be found in the dynamics of an electron driven in a solid by an external field. By taking the Bloch acceleration theorem

$$
k(t)=k_{0}-\frac{e}{\hbar} \int E(t) \mathrm{d} t,
$$

as the time-dependent electron wave number, where $A(t)$ is the vector potential and $k_{0}$ the initial electron wave number, and

$$
\alpha(t)=2 \hbar v_{\mathrm{F}} k(t)
$$




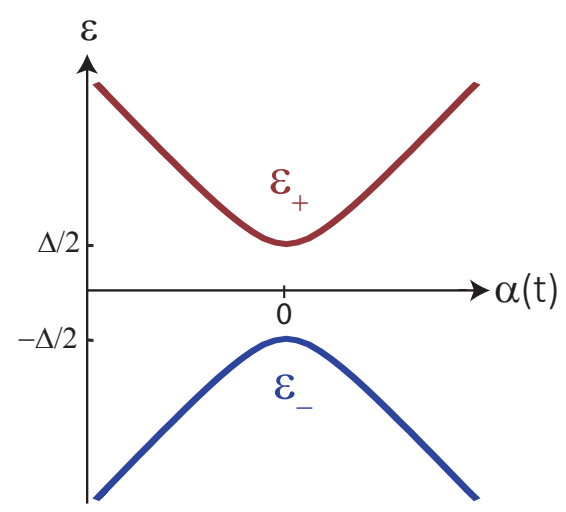

FIG. 4. Two-level system. Energy $\varepsilon$ versus timedependent energy bias $\alpha$. The blue and red solid lines represents the adiabatic energy basis of the two-level system with two instantaneous eigenstates $\varepsilon_{+}$and $\varepsilon_{-}$. For $\alpha=0$, the energy difference $\varepsilon(t)$ between the two eigenstates is given by $\Delta$.

as the time-dependent energy bias, with $v_{\mathrm{F}}$ the Fermi velocity, we can rewrite Eq. (10) to

$$
\hat{\mathcal{H}}(t)=\left(\begin{array}{cc}
-\frac{\Delta}{2} & \hbar v_{\mathrm{F}} k(t) \\
\hbar v_{\mathrm{F}} k(t) & \frac{\Delta}{2}
\end{array}\right) .
$$

For $k_{0}=0$ and a periodic oscillating electric field $E(t)=$ $E_{0} \sin (\omega t)$, Eq. 15 has the form as that of a driven twolevel Rabi system with

$$
\Omega_{\mathrm{R}}=\frac{v_{\mathrm{F}} e E_{0}}{\hbar \omega}
$$

the Rabi frequency. $\omega$ is the angular frequency and $E_{0}$ the peak electric field strength.

To obtain the temporal evolution of an electron in the two-level system, we assume that the electron dynamics is coherent and can thus be described by the timedependent Schrödinger equation (TDSE)

$$
i \hbar \frac{\partial \Psi\left(k_{0}, t\right)}{\partial t}=\hat{\mathcal{H}}(t) \Psi\left(k_{0}, t\right)
$$

with $\Psi\left(k_{0}, t\right)=\sum_{m=\mathrm{CB}, \mathrm{VB}} \beta^{(m)}\left(k_{0}, t\right) \Phi\left(k_{0}\right)$ the electron wave function with $\Phi$ the Bloch states of the field-free system and $\beta^{(m)}$ the expansion coefficients of Eq. (17) 53 .

\section{Keldysh adiabaticity parameter}

The general form of the Keldysh parameter for a twolevel system reads

$$
\gamma=\frac{\omega \sqrt{m \Delta}}{e E_{0}}
$$

with $m$ the effective electron mass [34]. Using Eqs. 12 and (14) we obtain

$$
m=\hbar^{2}\left[\left.\frac{\partial^{2} \varepsilon(k)}{\partial k^{2}}\right|_{k_{0}=0}\right]^{-1}=\frac{\Delta}{4 v_{\mathrm{F}}^{2}} .
$$

Applying the Rabi frequency [Eq. (16)], we rewrite the Keldysh adiabaticity parameter for an oscillating electric field

$$
\gamma=\frac{\omega \Delta}{2 v_{\mathrm{F}} e E_{0}}=\frac{\Delta}{2 \hbar \Omega_{\mathrm{R}}} .
$$

Here, the Keldysh adiabaticity parameter is given as the characteristic interaction strength $2 \hbar \Omega_{\mathrm{R}}$ and the band gap $\Delta$. Note that in case of resonant excitation $\hbar \omega=\Delta$ and $\omega \ll \Omega_{\mathrm{R}}$, this regime is known as carrier-wave Rabi flopping.

\section{Landau-Zener transition}

Following the framework of Landau-Zener (LZ) transitions, it is useful to work with a Hamiltonian $\hat{\mathcal{H}}^{\prime}(t)$ with time-dependent diagonal components, which can be achieved by applying the unitary transformation

$$
\hat{U}=\exp \left(i \frac{\pi}{4} \hat{\sigma}_{\mathrm{y}}\right)
$$

We obtain

$$
\begin{aligned}
\hat{\mathcal{H}}^{\prime}(t) & =\hat{U} \hat{\mathcal{H}}(t) \hat{U}^{-1} \\
& =-\frac{\Delta}{2} \hat{\sigma}_{\mathrm{x}}-\frac{\alpha}{2} \hat{\sigma}_{\mathrm{z}} .
\end{aligned}
$$

In the vicinity of an LZ transition we linearize $\alpha(t)$, i.e., $\frac{\cos (\omega t)}{\omega} \approx t$ and obtain $\alpha(t) \approx \alpha_{0} t$, with $\alpha_{0}=2 v_{\mathrm{F}} e E_{0}$. Now, $\hat{\mathcal{H}}^{\prime}(t)$ reads

$$
\hat{\mathcal{H}}^{\prime}(t)=-\frac{1}{2}\left(\begin{array}{cc}
\alpha_{0} t & \Delta \\
\Delta & -\alpha_{0} t
\end{array}\right) .
$$

This Hamiltonian has the form as a Hamiltonian for an avoided crossing model with the Landau-Zener transition probability

$$
P_{\mathrm{LZ}}=\exp \left(-2 \pi \delta_{\mathrm{LZ}}\right)
$$

with $\delta_{\mathrm{LZ}}=\Delta^{2} /(4 \hbar \alpha)$, the Landau-Zener adiabaticity parameter [21]. For $\delta_{\mathrm{LZ}} \ll 1, P_{\mathrm{LZ}} \rightarrow 1$ and an interband transition becomes likely.

\section{E. Analytic resonance condition}

When an electric field is applied to solids, the wave number $k(t)$ of an electron changes according to Eq. 13 . 
Within one optical cycle, the resulting intraband motion leads to the accumulation of a dynamical phase

$$
\phi=\frac{1}{\hbar} \int_{0}^{T_{0}} \varepsilon(t) \mathrm{d} t
$$

with $T_{0}=2 \pi / \omega$. We assume a periodically oscillating electric field to obtain an analytic expression for the resonance condition under the presence of intraband motion. Using the Keldysh parameter, we rewrite Eq. (12):

$$
\begin{aligned}
\varepsilon(t) & =\sqrt{\Delta^{2}+\left(2 v_{\mathrm{F}} e \frac{E_{0}}{\omega} \cos (\omega t)\right)^{2}} \\
& =\Delta \sqrt{1+\left(\frac{\cos (\omega t)}{\gamma}\right)^{2}} .
\end{aligned}
$$

Taking $\omega t=t^{\prime}$ and $t^{\prime \prime}=t^{\prime}-\frac{\pi}{2}$ we write

$$
\begin{aligned}
\phi & =\frac{2 \Delta}{\hbar \omega} \cdot \int_{0}^{\pi} \sqrt{1+\left(\gamma^{-1} \cos \left(t^{\prime}\right)\right)^{2}} \mathrm{~d} t^{\prime} \\
& =\frac{4 \Delta}{\hbar \omega} \cdot \int_{0}^{\frac{\pi}{2}} \sqrt{1+\left(\gamma^{-1} \sin \left(t^{\prime}\right)\right)^{2}} \mathrm{~d} t^{\prime \prime} .
\end{aligned}
$$

Now we apply the complete second order elliptic integral

$$
\phi=\frac{4 \Delta}{\hbar \omega} \mathfrak{e}_{l}\left(\gamma^{-2}\right) .
$$

When a phase of $\phi=2 \pi$ is accumulated, population at the next higher multi-photon resonance $(n)$ is found. Thus,

$$
\frac{4 \Delta}{\hbar \omega} \mathfrak{e}_{l}\left(\gamma^{-2}\right) \stackrel{!}{=} 2 \pi \cdot n
$$

needs to be fulfilled and the the condition for analytical resonance under the presence of intraband motion reads

$$
M=\frac{\pi n}{2 \mathfrak{e}_{l}\left(\gamma^{-2}\right)} .
$$

For $\gamma \gg 1$, the elliptic integral becomes $\frac{\pi}{2}$ and the photon resonances are found at multiples of the bandgap ( $M=$ $n)$.

\section{F. Genericity under pulse distortion and dephasing}

The categorization of excitation regimes in a stronglydriven two-level system is general and remains valid for different pulse durations, dispersion and dephasing, i.e., $z_{\mathcal{R}}, \delta_{\mathrm{LZ}}$ and $\gamma$ depends on the driving frequency, peak electric field strength and the energy spacing between the two bands. To prove this, we show in Fig. 5 the residual conduction band population for three different bandwidth-limited pulse durations (Fig. 5 a: $5 \mathrm{fs}, \mathrm{b}: 10 \mathrm{fs}$, c: $20 \mathrm{fs}$ ) centered at a photon energy of $\hbar \omega=1.55 \mathrm{eV}$. Increasing the pulse duration results in a decrease in the spectral width of the photon orders and an increase of
Rabi cycles as a function of $\gamma$, i.e., more Rabi cycles can be performed during the laser pulse. In the field-driven regimes, increasing the number of optical cycles results in more subsequent LZ-transitions and a different accumulated dynamical phase. In the adiabatic-impulsive LZS regime, both lead to strong mixing of states resulting in finer features in the residual conduction band population. Whereas the exact value of $\rho^{\mathrm{CB}}$ depends on the pulse shape, the categorization into different excitation regimes is determined by peak electric field strength.

Similarly, introducing dispersion does not affect the categorization. In Fig. 5b we show the residual conduction band population of the same pulse applied in Fig.5a, but stretched by $5 \mathrm{~mm} \mathrm{SiO}_{2}$ (GDD: $180.8 \mathrm{fs}^{2}$, TOD: $\left.137.3 \mathrm{fs}^{3}\right)$. The visibility of the Rabi-oscillations is decreased since the spectral components are delayed with respect to each other. In the field-driven regimes, all features are preserved while again, the exact value of conduction band population is changed due to different accumulation of dynamical phase.

Figure 6 shows the residual conduction band population for a pulse duration of $5 \mathrm{fs}$ including a dephasing time constant $T_{2}=3 \mathrm{fs} 23$. Decreasing the dephasing time results in an increase of $\rho^{\mathrm{CB}}$ in the adiabatic regime, i.e., transiently populated carriers remain after the laser pulse and the residual population in the adiabatic-impulsive LZS regime becomes smeared out. LZS interference becomes less important due to decoherence and the visibility of features decreases consequently. We note that the dephasing time constant $T_{2}$ can be understood as decay of quantum coherence in a coherently driven system.

\section{G. Relativistic regime}

The classical equation of motion of a free electron in an optical field is given by

$$
\frac{\mathrm{d} \mathbf{p}}{\mathrm{d} t}=-e(\mathbf{E}(\mathbf{x}, t)+\mathbf{v} \times \mathbf{B}(\mathbf{x}, t)),
$$

with peak magnetic field $\left|B_{0}\right|=E_{0} / c$ (c: speed of light, e: electron charge, p: electron momentum, v: electron velocity). In classical Newtonian mechanics we assume that $v \ll c$. This implies that the contribution of the second term in Eq. (31) is negligible. Assuming an oscillating electric field, the maximal velocity is $v_{\max }=e E_{0} /\left(\omega m_{e}\right)$. When $v_{\max }$ becomes comparable to $c$, the normalized vector potential

$$
a_{0}=\frac{e E_{0}}{\omega m_{e} c},
$$

reaches unity and relativistic effects become important [55. In case of a TLS, we replace the electron rest mass $m_{e}$ with the effective mass [Eq. [19]] and obtain

$$
a_{0, \mathrm{TLS}}=\frac{v_{\mathrm{F}}}{\gamma c} .
$$



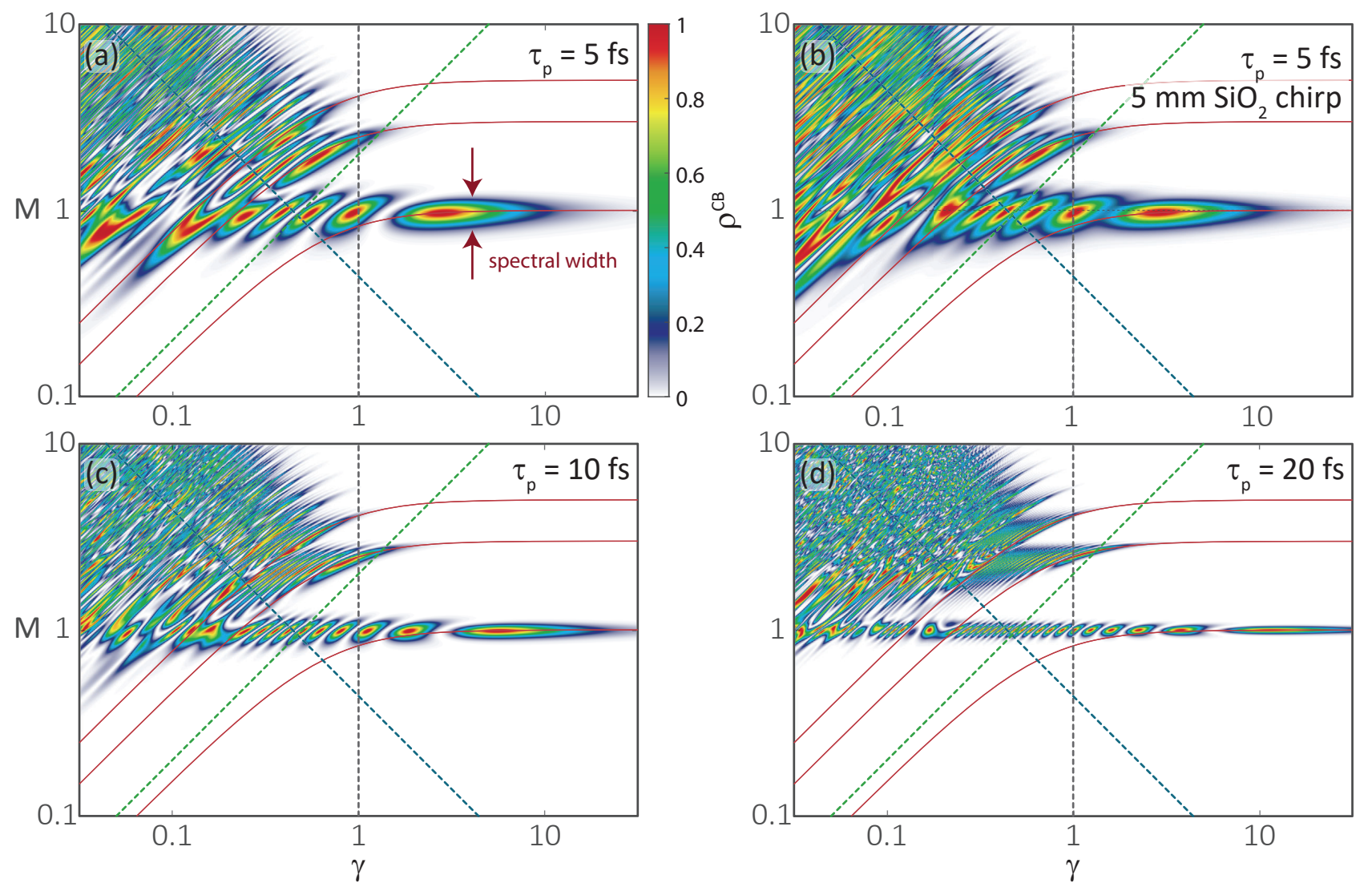

FIG. 5. Different pulse durations and dispersion. Map of the residual conduction band population for three different bandwidth-limited pulse durations ( $5 \mathrm{fs}(\mathrm{a}), 10 \mathrm{fs}(\mathrm{c}), 20 \mathrm{fs}(\mathrm{d})$ ) and for a $5 \mathrm{fs}$ pulse with dispersion of $5 \mathrm{~mm} \mathrm{SiO}_{2}$ (b). Whereas for $\gamma>1$ only perturbative, resonant one-photon $(n=1)$ absorption at $M=1$ is found, for $\gamma<1$ off-resonant excitation occurs. In particular for $P_{\mathrm{LZ}} \approx 0.5, \delta_{\mathrm{LZ}} \approx \ln (2) /(2 \pi)$ and $z_{\mathcal{R}}>1$, defining the adiabatic-impulsive regime (5), most off-resonant excitation is found. The red lines represent odd resonances for the light matter interaction. The dashed green line represents $z_{\mathcal{R}}=1$ and the dashed blue line $P_{\mathrm{LZ}} \approx 0.5$. See main text for detailed discussion.

The relativistic regime $\left(a_{0}>1\right)$ is entered for $\gamma<\frac{v_{F}}{c}$. Hence, in the TLS considered here with $v_{F}=1 \mathrm{~nm} / \mathrm{fs}$, the magnetic component of the optical field can be neglected for $\gamma>0.007$. Figure 7 shows the full $\gamma$-M map including the relativistic regime.

\section{ACKNOWLEDGMENTS}

This work has been supported in part by the European Research Council (Consolidator Grant "NearFieldAtto"), Deutsche Forschungsgemeinschaft (Sonderforschungsbereich 953 "Synthetic Carbon Allotropes", project 182849149) and the PETACom project financed by Future and Emerging Technologies Open H2020 program. P. H. greatefully acknowledges a Fellowship from Max Planck Institute of the Science of Light (MPL). We thank Vladislav S. Yakovlev for discussions.
[1] P.B. Corkum and F. Krausz, "Attosecond science," Nature Physics 3, 381-387 (2007).

[2] Shambhu Ghimire and David A. Reis, "High-harmonic generation from solids," Nature Physics 15, 10-16 (2019).

[3] G. Vampa, T. J. Hammond, N. Thiré, B. E. Schmidt, F. Légaré, C. R. McDonald, T. Brabec, and P. B. Corkum, "Linking high harmonics from gases and solids,"
Nature 522, 462-464 (2015)

[4] P. Jürgens, B. Liewehr, B. Kruse, C. Peltz, D. Engel, A. Husakou, T. Witting, M. Ivanov, M. J.J. Vrakking, T. Fennel, and A. Mermillod-Blondin, "Origin of strongfield-induced low-order harmonic generation in amorphous quartz," Nature Physics (2020). 


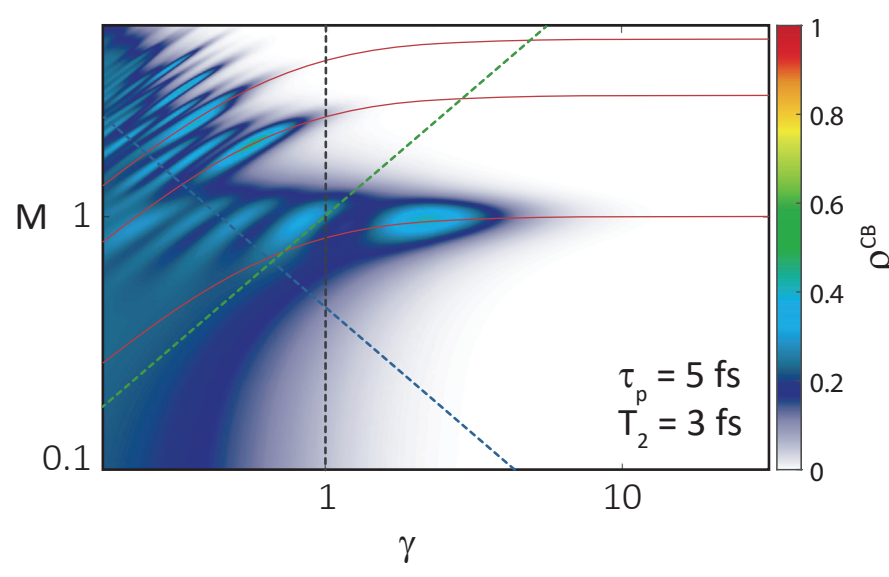

FIG. 6. Dephasing of population. Map of the residual conduction band population assuming a dephasing time constant of $T_{2}=3 \mathrm{fs}$ and a pulse duration of $5 \mathrm{fs}$. The red lines represent odd resonances for the light matter interaction. The dashed green line represents $z_{\mathcal{R}}=1$ and the dashed blue line $P_{\mathrm{LZ}} \approx 0.5$. See main text for detailed discussion.

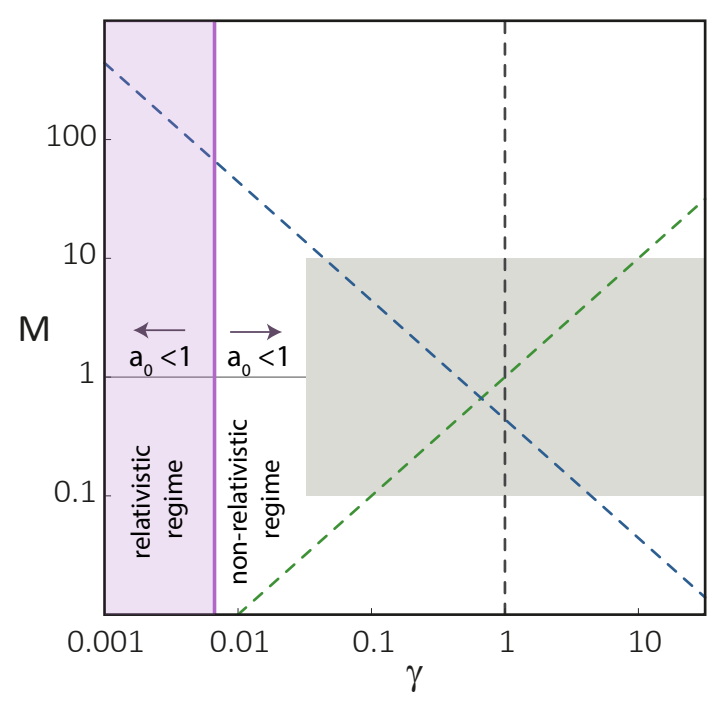

FIG. 7. Relativistic regime in the strongly-driven TLS. The gray box represents the regimes discussed in this paper. Relativistic effects become important when the normalized vector potential $a_{0}$ becomes smaller than 1 . In the TLS system considered here, a Keldysh parameter smaller than 0.007 is required to enter the relativistic regime.

[5] M. F. Ciappina, J. A. Pérez-Hernández, A. S. Landsman, W. A. Okell, S. Zherebtsov, B. Förg, J. Schötz, L. Seiffert, T. Fennel, T. Shaaran, T. Zimmermann, A. Chacón, R. Guichard, A. Zaïr, J.W.G. Tisch, J.P. Marangos, T. Witting, Braun A., S.A. Maier, L. Roso, M. Krüger, P. Hommelhoff, M.F Kling, F. Krausz, M. Lewenstein, and Report O N Progress, "Attosecond physics at the nanoscale," Reports on Progress in Physics 80, 054401 (2017).
[6] Agustin Schiffrin, Tim Paasch-Colberg, Nicholas Karpowicz, Vadym Apalkov, Daniel Gerster, Sascha Mühlbrandt, Michael Korbman, Joachim Reichert, Martin Schultze, Simon Holzner, Johannes V. Barth, Reinhard Kienberger, Ralph Ernstorfer, Vladislav S. Yakovlev, Mark I. Stockman, and Ferenc Krausz, "Optical-field-induced current in dielectrics," Nature 493, 70-74 (2013)

[7] Hamed Koochaki Kelardeh, Vadym Apalkov, and Mark I. Stockman, "Graphene in ultrafast and superstrong laser fields," Physical Review B 91, 045439 (2015).

[8] Takuya Higuchi, Christian Heide, Konrad Ullmann, Heiko B. Weber, and Peter Hommelhoff, "Light-fielddriven currents in graphene," Nature 550, 224-228 (2017)

[9] Anton Frisk Kockum, Adam Miranowicz, Simone De Liberato, Salvatore Savasta, and Franco Nori, "Ultrastrong coupling between light and matter," Nature Reviews Physics 19, 19-40 (2019).

[10] P. Forn-Díaz, L. Lamata, E. Rico, J. Kono, and E. Solano, "Ultrastrong coupling regimes of light-matter interaction," Reviews of Modern Physics 91, 25005 (2019).

[11] D. M. Berns, W. D. Oliver, S. O. Valenzuela, A. V. Shytov, K. K. Berggren, L. S. Levitov, and T. P. Orlando, "Coherent quasiclassical dynamics of a persistent current qubit," Physical Review Letters 97, 150502 (2006).

[12] Ruben M. Otxoa, Anasua Chatterjee, Sergey N. Shevchenko, Sylvain Barraud, Franco Nori, and M.F. Gonzalez-Zalba, "Quantum interference capacitor based on double-passage Landau-Zener-Stückelberg-Majorana interferometry," Physical Review B 100, 205425 (2019).

[13] J. Reimann, S. Schlauderer, C. P. Schmid, F. Langer, S. Baierl, K. A. Kokh, O. E. Tereshchenko, A. Kimura, C. Lange, J. Güdde, U. Höfer, and R. Huber, "Subcycle observation of lightwave-driven Dirac currents in a topological surface band," Nature 562, 396-400 (2018).

[14] S. Azar Oliaei Motlagh, Fatemeh Nematollahi, Vadym Apalkov, and Mark I. Stockman, "Topological resonance and single-optical-cycle valley polarization in gapped graphene," Physical Review B 100, 115431 (2019)

[15] C. Vaswani, L.-L. Wang, D. H. Mudiyanselage, Q. Li, P. M. Lozano, G. D. Gu, D. Cheng, B. Song, L. Luo, R. H. J. Kim, C. Huang, Z. Liu, M. Mootz, I. E. Perakis, Y. Yao, K. M. Ho, and J. Wang, "Light-Driven Raman Coherence as a Nonthermal Route to Ultrafast Topology Switching in a Dirac Semimetal," Physical Review X 10, 021013 (2020).

[16] Wei Nie, Z. H. Peng, Franco Nori, and Yu Xi Liu, "Topologically Protected Quantum Coherence in a Superatom," Physical Review Letters 124, 23603 (2020).

[17] Leslie Allen and Joseph H Eberly, Optical resonance and two-level atoms, (Dover Publications, 1987).

[18] Claude Cohen-Tannoudji, Jacques Dupont-Roc, and Gilbert Grynberg, Atom-photon interactions: basic processes and applications (Wiley-VCH, 2004).

[19] S. Ashhab, J. R. Johansson, A. M. Zagoskin, and Franco Nori, "Two-level systems driven by large-amplitude fields," Physical Review A - Atomic, Molecular, and Optical Physics 75, 063414 (2007).

[20] Kenichi L. Ishikawa, "Nonlinear optical response of graphene in time domain," Physical Review B 82, 201402(R) (2010) 
[21] Sergey N. Shevchenko, S. Ashhab, and Franco Nori, "Landau-Zener-Stückelberg interferometry," Physics Reports 492, 1-30 (2010)

[22] Larisa A Chizhova, Florian Libisch, and Joachim Burgdörfer, "Nonlinear response of graphene to a fewcycle terahertz laser pulse: Role of doping and disorder," Physical Review B 94, 075412 (2016).

[23] Michael S. Wismer, Stanislav Yu Kruchinin, Marcelo Ciappina, Mark I. Stockman, and Vladislav S. Yakovlev, "Strong-Field Resonant Dynamics in Semiconductors," Physical Review Letters 116, 197401 (2016).

[24] Francois Fillion-Gourdeau, Denis Gagnon, Catherine Lefebvre, and Steve Maclean, "Time-domain quantum interference in graphene," Physical Review B 94, 125423 (2016).

[25] S.Y. Kruchinin, F. Krausz, and V.S. Yakovlev, "Colloquium : Strong-field phenomena in periodic systems," Reviews of Modern Physics 90, 21002 (2018)

[26] Shunsuke A. Sato, Matteo Lucchini, Mikhail Volkov, Fabian Schlaepfer, Lukas Gallmann, Ursula Keller, and Angel Rubio, "Role of intraband transitions in photocarrier generation," Physical Review B 98, 035202 (2018).

[27] Q. Z. Li, P. Elliott, J. K. Dewhurst, S. Sharma, and S. Shallcross, "Ab-intio study of ultrafast charge dynamics in graphene," (2020), arXiv:2012.00435.

[28] Takuya Higuchi, Mark I. Stockman, and Peter Hommelhoff, "Strong-field perspective on high-harmonic radiation from bulk solids," Physical Review Letters 113, 213901 (2014)

[29] T. T. Luu, M. Garg, S. Yu. Kruchinin, A. Moulet, M. Th Hassan, and E. Goulielmakis, "Extreme ultraviolet highharmonic spectroscopy of solids," Nature 521, 498-502 (2015)

[30] Hanzhe Liu, Yilei Li, Yong Sing You, Shambhu Ghimire, Tony F. Heinz, and David A. Reis, "High-harmonic generation from an atomically thin semiconductor," Nature Physics 13, 262-265 (2017).

[31] Nicolas Tancogne-Dejean, Oliver D. Mücke, Franz X. Kärtner, and Angel Rubio, "Ellipticity dependence of high-harmonic generation in solids originating from coupled intraband and interband dynamics," Nature Communications 8, 745 (2017)

[32] Tran Trung Luu, Zhong Yin, Arohi Jain, Thomas Gaumnitz, Yoann Pertot, Jun Ma, and Hans Jakob Wörner, "Extreme-ultraviolet high-harmonic generation in liquids," Nature Communications 9, 3723 (2018)

[33] Keisuke Kaneshima, Yasushi Shinohara, Kengo Takeuchi, Nobuhisa Ishii, Kotaro Imasaka, Tomohiro Kaji, Satoshi Ashihara, Kenichi L. Ishikawa, and Jiro Itatani, "Polarization-Resolved Study of High Harmonics from Bulk Semiconductors," Physical Review Letters 120, 243903 (2018).

[34] L. V. Keldysh, "Ionization in the field of a strong electromagnetic wave," Sov. Phys. JETP 20, 1307-1314 (1965).

[35] Howard R. Reiss, "Effect of an intense electromagnetic field on a weakly bound system," Physical Review A 22, 1786-1813 (1980)

[36] Martin Wegener and Extreme Nonlinear Optics, Extreme Nonlinear Optics (Springer Science \& Business Media, 2005).

[37] L D Landau, "Zur Theorie der Energieubertragung II," Phys. Z. Sowjetunion 2, 46-51 (1932)

[38] Clarence Zener, "Non- Adiabatic Crossing of Energy Levels." Proceedings of the Royal Society of London A 137,
696-702 (1932)

[39] Yosuke Kayanuma, "Stokes phase and geometrical phase in a driven two-level system," Physical Review A Atomic, Molecular, and Optical Physics 55, R2495R2498 (1997)

[40] Felix Bloch, "Über die Quantenmechanik der Elektronen in Kristallgittern," Zeitschrift für Physik 52, 555-600 (1929)

[41] Larisa A Chizhova, Florian Libisch, and Joachim Burgdörfer, "High-harmonic generation in graphene: Interband response and the harmonic cutoff," Physical Review B 95, 085436 (2017).

[42] Jan R. Rubbmark, Michael M. Kash, Michael G. Littman, and Daniel Kleppner, "Dynamical effects at avoided level crossings: A study of the Landau-Zener effect using Rydberg atoms," Physical Review A 23, 31073117 (1981)

[43] Moshe Shapiro, Marc J.J. Vrakking, and Albert Stolow, "Nonadiabatic wave packet dynamics: Experiment and theory in IBr," Journal of Chemical Physics 110, 24652473 (1999).

[44] G. Vampa, C. R. McDonald, G. Orlando, D. D. Klug, P. B. Corkum, and T. Brabec, "Theoretical analysis of high-harmonic generation in solids," Physical Review Letters 113, 073901 (2014).

[45] Ignacio Franco and Paul Brumer, "Minimum requirements for laser-induced symmetry breaking in quantum and classical mechanics," Journal of Physics B: Atomic, Molecular and Optical Physics 41, 074003 (2008).

[46] Andreas Bayer, Marcel Pozimski, Simon Schambeck, Dieter Schuh, Rupert Huber, Dominique Bougeard, and Christoph Lange, "Terahertz Light - Matter Interaction beyond Unity Coupling Strength," Nano Letters 17, 6340-6344 (2017).

[47] M. Lezius, V. Blanchet, Misha Yu Ivanov, and Albert Stolow, "Polyatomic molecules in strong laser fields: Nonadiabatic multielectron dynamics," Journal of Chemical Physics 117, 1575-1588 (2002).

[48] H J Wörner, J B Bertrand, B Fabre, J Higuet, H Ruf, A Dubrouil, S Patchkovskii, M Spanner, Y Mairesse, V Blanchet, E Mével, E Constant, P B Corkum, and D M Villeneuve, "Conical Intersection Dynamics in NO2 Probed by Homodyne High-Harmonic Spectroscopy," Science 334, 208 LP - 212 (2011).

[49] Matthias F. Kling, Philipp Von Den Hoff, Irina Znakovskaya, and Regina De Vivie-Riedle, "(Sub)femtosecond control of molecular reactions via tailoring the electric field of light," Physical Chemistry Chemical Physics 15, 9448-9467 (2013)

[50] Gregory H. Wannier, "Wave functions and effective hamiltonian for bloch electrons in an electric field," Physical Review 117, 432-439 (1960)

[51] Bogdan Damski, "The simplest quantum model supporting the kibble-zurek mechanism of topological defect production: Landau-zener transitions from a new perspective," Physical Review Letters 95, 035701 (2005).

[52] S.A. OliaeiMotlagh, J.S. Wu, Vadym Apalkov, and Mark I. Stockman, "Femtosecond valley polarization and topological resonances in transition metal dichalcogenides," Physical Review B 98, 081406(R) (2018).

[53] S. Azar Oliaei Motlagh, Fatemeh Nematollahi, Aranyo Mitra, Ahmal Jawad Zafar, Vadym Apalkov, and Mark I. Stockman, "Ultrafast optical currents in gapped 
graphene," Journal of Physics Condensed Matter 32, 065305 (2020).

[54] Iulia Buluta, Sahel Ashhab, and Franco Nori, "Natural and artificial atoms for quantum computation," Reports on Progress in Physics 74, 104401 (2011)

[55] M. Fuchs, R. Weingartner, A. Popp, Z. Major, S. Becker, J. Osterhoff, I. Cortrie, B. Zeitler, R. Hörlein, G. D. Tsakiris, et al., Nature Physics 5, 826 (2009). 